\title{
Discovery of a Jetlike Structure at the High- Redshift QSO CXOMP J084128.3+131107
}

\section{Citation}

Schwartz, D. A., J. Silverman, M. Birkinshaw, M. Karovska, T. Aldcroft, W. Barkhouse, P. Green, D.-W. Kim, B. J. Wilkes, and D. M. Worrall. 2004. "Discovery of a Jetlike Structure at the HighRedshift QSO CXOMP J084128.3+131107." The Astrophysical Journal 605 (2) (March 29): L105L108. doi:10.1086/420843.

\section{Published Version}

doi:10.1086/420843

\section{Permanent link}

http://nrs.harvard.edu/urn-3:HUL.InstRepos:30212164

\section{Terms of Use}

This article was downloaded from Harvard University's DASH repository, and is made available under the terms and conditions applicable to Other Posted Material, as set forth at http:// nrs.harvard.edu/urn-3:HUL.InstRepos:dash.current.terms-of-use\#LAA

\section{Share Your Story}

The Harvard community has made this article openly available.

Please share how this access benefits you. Submit a story.

\section{Accessibility}




\title{
DISCOVERY OF A JETLIKE STRUCTURE AT THE HIGH-REDSHIFT QSO CXOMP J084128.3+131107
}

\author{
D. A. Schwartz, J. Silverman, M. Birkinshaw, ${ }^{1}$ M. Karovska, T. Aldcroft, W. Barkhouse, \\ P. Green, D.-W. Kim, B. J. Wilkes, AND D. M. Worrall ${ }^{1}$ \\ Harvard-Smithsonian Center for Astrophysics, 60 Garden Street, Cambridge, MA 02138; das@ head.cfa.harvard.edu \\ Received 2003 December 4; accepted 2004 March 4; published 2004 March 29
}

\begin{abstract}
The Chandra Multiwavelength Project has discovered a jetlike structure associated with a newly recognized QSO at redshift $z=1.866$. The system was 9.4 off-axis during an observation of 3C 207. Although significantly distorted by the mirror point-spread function, we use both a ray trace and a nearby bright point source to show that the X-ray image must arise from some combination of point and extended sources, or else from a minimum of three distinct point sources. We favor the former situation, as three unrelated sources would have a small probability of occurring by chance in such a close alignment. We show that interpretation as a jet emitting Xrays via inverse Compton scattering on the cosmic microwave background is plausible. This would be a surprising and unique discovery of a radio-quiet QSO with an X-ray jet, since we have obtained upper limits of $100 \mu \mathrm{Jy}$ on the QSO emission at $8.46 \mathrm{GHz}$ and limits of $200 \mu \mathrm{Jy}$ for emission from the putative jet.
\end{abstract}

Subject headings: galaxies: jets — quasars: general — X-rays: galaxies

\section{INTRODUCTION}

The objectives of the Chandra Multiwavelength Project (ChaMP) include identification and categorization of a complete well-defined sample of serendipitous sources (Kim et al. 2004; Green et al. 2004). The results will be of use, e.g., to study luminosity functions and their evolution, to quantify the newly resolved source(s) of the hard diffuse X-ray background, and to study cosmic structure and clustering of active galactic nuclei and galaxies. The wide angle nature of this survey also makes it ideal for discovering rare and unusual objects suitable for detailed study, e.g., lensed QSOs and X-ray jets.

Schwartz (2002a, 2002b) has pointed out that if the jets observed in X-rays on scales of tens to hundreds of kiloparsecs are emitting via inverse Compton (IC) scattering of the cosmic microwave background (CMB), as suggested by Tavecchio et al. (2000) and Celotti et al. (2001), then they will maintain the same apparent surface brightness independent of redshift and therefore can be detected to arbitrarily large redshifts, up to the epoch at which they form. The Chandra observations of such large-scale jets in QSOs and powerful FR II radio sources are typically interpreted as IC/CMB emission (Schwartz et al. 2000; Harris \& Krawczynski 2002; Marshall et al. 2001; Sambruna et al. 2001; Siemiginowska et al. 2002). All such interpretations require the assumption either that the jet is relativistically beamed with Doppler factors of the order of $\delta \sim 3-15$ or that the energy density in relativistic electrons grossly exceeds the magnetic field energy density by at least 2 orders of magnitude. Detection of the X-ray "beacons" predicted by Schwartz (2002a, 2002b) would provide additional evidence that the above assumptions are well founded.

We report the discovery of a candidate for such a system: CXOMP J084128.3+131107 (hereafter called J0841). The Xray image shows an elongated structure. Despite the broad point-spread function (PSF) of the Chandra telescope at this 9.4 off-axis angle, we show that at least three point sources would be required to simulate the observed extent. We favor an interpretation of emission from the jet of an optically identified QSO that is close to the peak X-ray intensity. We also

\footnotetext{
${ }^{1}$ Also at the Department of Physics, University of Bristol, Tyndall Avenue, Bristol BS8 1TL, UK.
}

mention alternate interpretations. Because of the small probability for three unrelated sources to occur by chance in this configuration, such interpretations may be even more unusual.

\section{OBSERVATIONS OF J0841}

The serendipitous detection of J0841 on the ACIS-I2 chip occurred using the data from observation ID (ObsID) 2130, an observation of 3C 207 with ACIS-S3 (Brunetti et al. 2002). Figure 1 shows the X-ray contours superposed on a red-band image. The strongest X-ray peak is coincident within 1.5 with an $r^{\prime}=20.9$ mag object. A spectrum of this object (Fig. 2) was obtained in a 10 minute exposure on Magellan using the lowdispersion survey spectrograph LDSS-2 and clearly shows a broad emission line QSO. The optical data have about $13 \AA$ resolution. The spectrum was cross-correlated against the composite Sloan Digital Sky Survey QSO spectrum (Vanden Berk 2001) to give a redshift 1.866 .

Although the contours in Figure 1 seem to indicate an extended $\mathrm{X}$-ray structure, one must be careful because of the distorted telescope response at this large off-axis angle. Figure 3 shows the X-ray data in the region of the QSO, together with data around the nearby Einstein medium survey point source MS 0838.6+1325 (Maccacaro et al. 1991), a $z=0.723$ QSO, also called EMSS 0841+131, which happens to lie in the same Chandra field at a similar off-axis angle, 9 '3 , and at the nearby azimuth of $247^{\circ}$ versus $265^{\circ}$ for J0841. Each is compared with a highfidelity ray trace ${ }^{2}$ of a $1.5 \mathrm{keV}$ point source at this off-axis angle and the same azimuth as J0841. ${ }^{3}$ Both QSOs are expected to have relatively hard spectra, for which $1.5 \mathrm{keV}$ is a good mean energy, so we do not expect significant effects due to spectral differences. J0841 is clearly not a single point source.

We now show that two point sources could not produce the observed X-ray structure. Specifically, in the top panel of Figure 3, taking point sources at the QSO position and at the center of the ellipse marked B, we show that region A contains a significant excess of counts over background plus those

\footnotetext{
${ }^{2}$ See http://cxc.harvard.edu/chart.

${ }^{3}$ Note that the Chandra point response function is azimuthally asymmetric; see http://cxc.harvard.edu/ccw_02.
} 


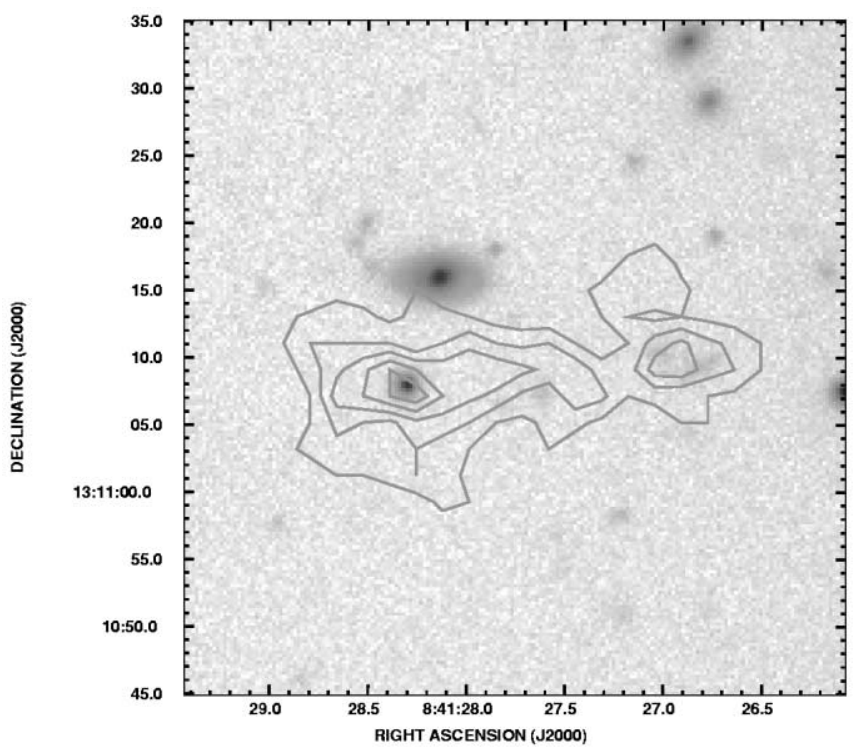

FIG. 1.-X-ray contours $(0.5-7 \mathrm{keV})$ in the region of J0841, superposed on a red-band image. Contour levels are $0.25,0.50,0.75,1.0$, and 1.25 counts $0 " 98 \times 00^{\prime \prime} 98 \mathrm{pixel}^{-1}$. Background is 0.03 counts pixel $^{-1}$. The $r^{\prime}=20.9$ object in the eastern contour peak is a QSO at redshift $z=1.866$. The position difference between the X-ray peak and the optical source is 1 1.5, consistent with the Chandra PSF distortion at this large off-axis angle.

counts that could be attributed to the QSO, plus those counts that could be attributed to the source B. The expected counts in box $\mathrm{A}$ are based on the measured ratio of counts in the ellipse marked QSO to the counts in a box marked A to the west of the QSO, or a similar box to the east of the QSO (not shown). We derive this predicted ratio both from real data, EMSS $0841+1314$, and from a ray trace, and in both cases we predict $\leq 10$ counts in box A (including the non-X-ray background). However, we observe 21 counts in box $\mathrm{A}$, and the probability of this is less than 0.1 .

We present the expected number of counts in box A in more detail for both methods: based on the ray-trace image (Fig. 3, middle panel) and based on the observation of EMSS 0841+131 (Fig. 3, bottom panel). The ray trace contains 1567 counts in box A and 29,945 in the QSO ellipse, for a measured ratio of 0.052 . For EMSS $0841+131$, after background subtraction, those numbers are 28.3 and 669.6 , for a ratio of $0.042 \pm$ 0.008 , consistent with the ray-trace prediction. From the observed 78.6 net counts inside the J0841 QSO ellipse (Fig. 3, top panel), after background subtraction, we use the ray-trace result of 0.052 to predict that 4.1 counts from the quasar would fall in box A. We do a similar analysis but with the ray trace or EMSS $0841+131$ source centered in the B region. We predict 0.113 and $0.091 \pm 0.012$, respectively, for the ray trace and for the EMSS $0841+131$ data, for the fraction of counts inside the B region that would appear in the box A. From the net 32.6 counts observed inside region B (Fig. 3, top panel), after background subtraction, the ray trace predicts an additional 3.7 counts in region A because of the point source in region B. Thus for region A in the top panel of Figure 3, we measure 21 counts and predict 7.8 from the putative point sources QSO and B, plus 1.7 background counts. The probability of observing 21 or more when 9.5 are expected is $0.086 \%$. We conclude that a minimum of three point sources would be needed if J0841 does not have extended X-ray emission.

The ellipses drawn in Figure 3 are 7" $\times$ 4".2 and are a contour

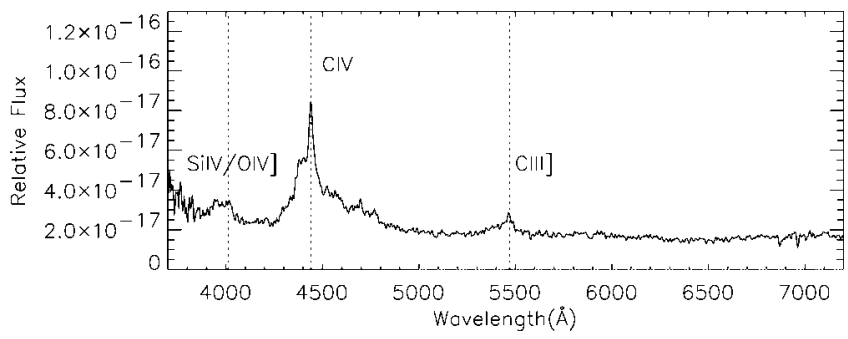

FIG. 2.-Ten minute Magellan exposure of J0841. The broad emission lines give a redshift $1.8661 \pm 0.0005$.

of $62 \%$ encircled energy based on EMSS $0841+131$ or $55 \%$ encircled energy based on the ray trace. The differences in these numbers are consistent with the statistics. For this type of analysis we could have drawn any particular curve around the QSO core - the particular ellipse chosen was convenient but arbitrary. The (unknown) true number of counts is not relevant: we can predict that the contributions to box A from a true total point-source flux are only about $2.9 \%$ and $6.2 \%$ from the west and east, respectively.

There are about 100 sources $\mathrm{deg}^{-2}$ above a flux of $10^{-14}$ ergs $\mathrm{cm}^{-2} \mathrm{~s}^{-1}$ (Giacconi et al. 2001). So there is a $2 \%$ chance that an unrelated source such as B could occur within $30^{\prime \prime}$ of the QSO. There is then only about a $0.3 \%$ chance of an independent third source appearing in a $10^{\prime \prime} \times 30^{\prime \prime}$ region between the first two sources. If we have three point sources, the probability is $\leq 6 \times 10^{-5}$ that they are unrelated. However, the ChaMP survey will eventually find of the order of $10^{3}$ QSOs brighter than $r^{\prime}=21$, so there might be as large as $10 \%$ prob-

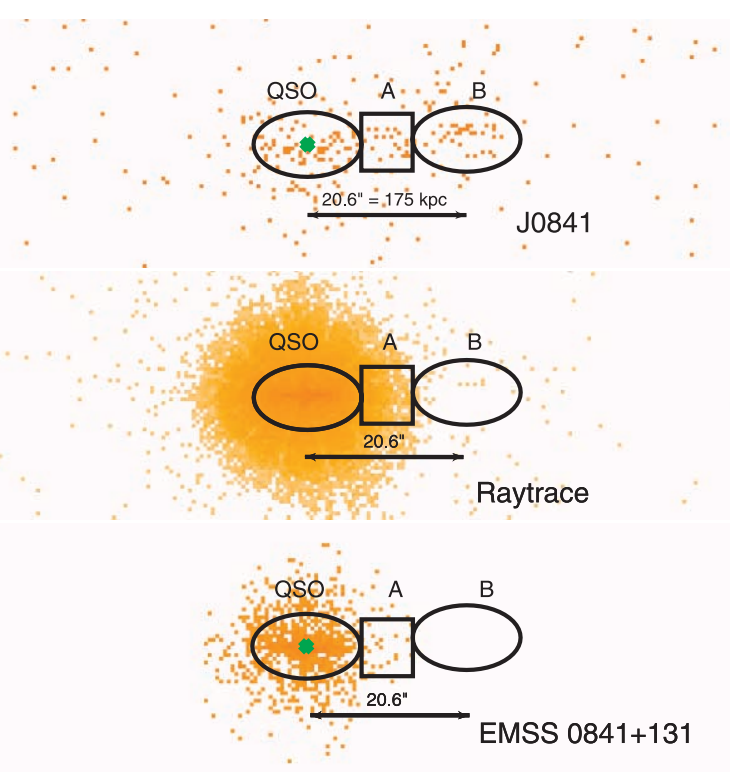

FIG. 3.-X-ray images $(0.5-7 \mathrm{keV})$ in 0 ".49 bins. Top to bottom: J0841 system, a ray-trace image of a $1.5 \mathrm{keV}$ point source at the off-axis position of J0841, and a point source, EMSS $0841+1314$, from the same observation as J0841. The ellipses labeled QSO are centered on the QSOs (green crosses, top and bottom) and the ray-trace axis (middle). The ellipse marked B is placed on the centroid of counts associated with the concentration $10^{\prime \prime}$ above the right arrow in the top panel. It is then placed in the same relative position to the ray-trace axis and EMSS $0841+1314$, in the middle and bottom panels. We show that box A in the top panel has excess counts and therefore represents a third source, based on ratios of the counts inside the QSO ellipses to those inside the box $\mathrm{A}$ in the bottom two panels (see text). 
ability for one such system of unrelated point sources to be found.

\section{INTERPRETATION AS AN X-RAY JET}

In Figure 3, we interpret the 78.6 net counts measured in the region indicated QSO as from the QSO core, and the 32.6 counts in region $\mathrm{B}$ and the net 11.5 from region $\mathrm{A}$ as from the jet. The ellipses shown are 55\% encircled energy regions, based on the ray-trace result, giving an inferred total counts of 143 from the QSO and 80 from the jet. This total of 223 inferred counts compares with 275 counts measured in a $25^{\prime \prime}$ radius circle about the QSO, the area of which contains an expected 73.7 background counts. The observation duration was 37,542 s (ObsID 2130 of 3C 207). Taking a conversion of $6 \times 10^{-12} \mathrm{ergs} \mathrm{cm}^{-2}$ $\mathrm{s}^{-1} /\left(\right.$ count $\mathrm{s}^{-1}$ ) (appropriate for an X-ray spectral energy index $\alpha=0.7$, and the measured Galactic absorption $n_{\mathrm{H}}=5 \times$ $10^{20} \mathrm{~cm}^{-2}$ [Stark et al. 1992]) gives estimated measured fluxes of $2.3 \times 10^{-14} \mathrm{ergs} \mathrm{cm}^{-2} \mathrm{~s}^{-1}$ for the QSO and $1.3 \times 10^{-14}$ ergs $\mathrm{cm}^{-2} \mathrm{~s}^{-1}$ for the jet, in the $0.5-7 \mathrm{keV}$ band. At $z=$ 1.866 this gives luminosities ${ }^{4}$ of $5.7 \times 10^{44} \mathrm{ergs} \mathrm{s}^{-1}$ for the QSO and $3.2 \times 10^{44} \mathrm{ergs} \mathrm{s}^{-1}$ for the jet. The roughly $20^{\prime \prime}$ length of the jet on the sky corresponds to a minimum length of $170 \mathrm{kpc}$ at the redshift $z=1.866$. Dividing the spectral data into six bins from 1 to $5 \mathrm{keV}$ and fixing the Galactic absorption, we can estimate an X-ray power-law energy index of $0.3 \pm$ 0.3 for the QSO and $0.5 \pm 0.3$ for the jet region.

We made a $1 \mathrm{hr}$ Very Large Array observation in the $\mathrm{C}$ array at $8.46 \mathrm{GHz}$ on 2003 January 10 and found no emission from the QSO to a $3 \sigma \mathrm{rms}$ noise limit of $100 \mu \mathrm{Jy},{ }^{5}$ or from the jet to a limit $200 \mu \mathrm{Jy}$. The broadband spectral indexes are $\alpha_{\text {ox }}=1.43$, and $\alpha_{\text {ro }}<0.04$, making it radio quiet, with a normal X-ray to optical ratio. Although it would be extremely surprising and unprecedented for a radio-quiet QSO to have a jet, it can be reasonably interpreted if the jet is highly beamed toward our line of sight and if the X-rays are being produced by IC scattering on the CMB. This is due to the extra factor of $\delta^{1+\alpha}$ (Dermer \& Schlickeiser 1994) by which the X-rays are boosted relative to the radio synchrotron emission, where the bulk relativistic Doppler factor $\delta$ is $[\Gamma(1-\beta \cos \theta)]^{-1}$, with $\Gamma$ being the Lorentz factor of the emitting region that is moving with a velocity $\beta c$ at an angle $\theta$ toward our line of sight. The spectral energy index is $\alpha$, where flux density is proportional to $\nu^{-\alpha}$. Tavecchio et al. (2000) and Celotti et al. (2001) showed how this effect could explain the surprisingly large X-ray flux observed from the PKS 0637-752 jet.

Figure 4 applies the analysis of Tavecchio et al. (2000). Here the lines with $\delta \propto 1 / B$ show the loci of equipartition between the magnetic fields and particles in the jet rest frame. We assume an electron population, $n(\gamma) \propto \gamma^{-m}$, with spectral index $m=2 \alpha+1=2.4$ producing radio emission between $10^{6}$ and $10^{12} \mathrm{~Hz}$ and with an equal energy density in protons. We consider the emitting volume as a cylinder of length $16^{\prime \prime} .3$. We do not resolve the width of the cylinder, and we take the radius to be the 2 ". 1 semiminor axis of the $62 \%$ encircled energy ellipse. The lines with $\delta \propto B$ show the loci for which the same electron population giving the radio emission produces the $\mathrm{X}$ rays by $\mathrm{IC} / \mathrm{CMB}$. The intersection of the solid lines give a solution for $B$ and $\delta$ in the case that the jet flux is at its limit

\footnotetext{
${ }^{4}$ We use $H_{0}=71 \mathrm{~km} \mathrm{~s}^{-1} \mathrm{Mpc}^{-1}$ and a flat accelerating universe with $\Omega_{0}=0.27$ and $\Omega_{\Lambda}=0.73$.

${ }^{5}$ See http://www.star.bris.ac.uk/ mb1/j0841.html.
}

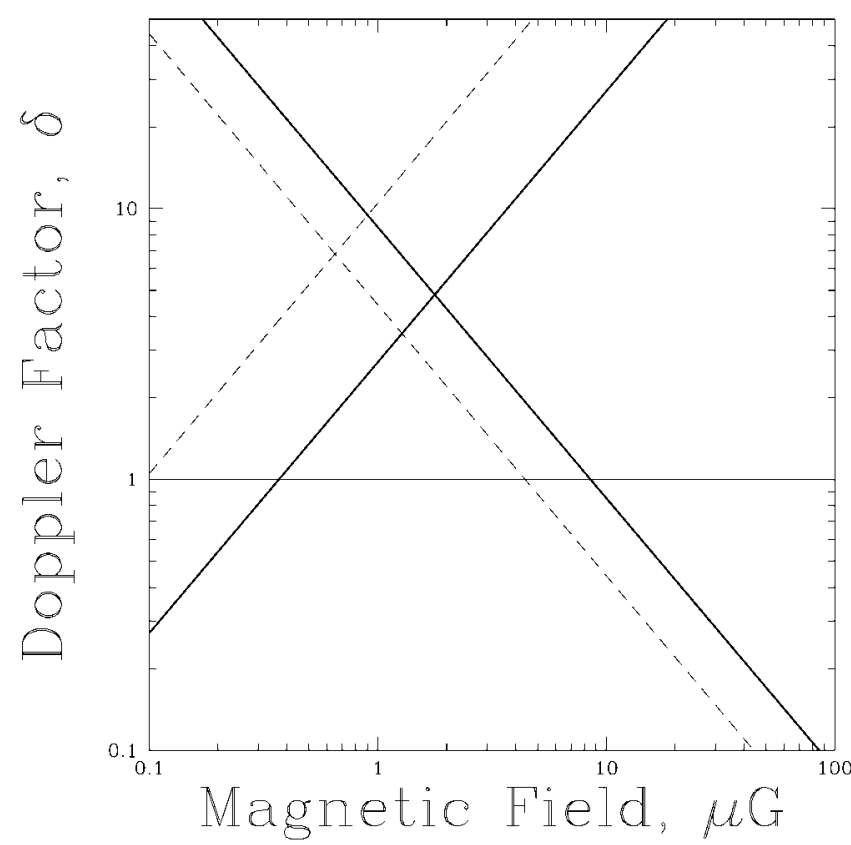

FIG. 4.- - Loci of equipartition $(\delta \propto 1 / B)$ and of X-ray emission via IC/CMB $(\delta \propto B)$ in the cases in which the $8.46 \mathrm{GHz}$ flux of the jet is at its upper limit of $200 \mu \mathrm{Jy}$ (solid lines) or 10 times weaker (dashed lines). The intersection of solid (or dashed) lines gives a solution for the rest-frame magnetic field and the Doppler factor.

of $200 \mu \mathrm{Jy}$ at $8.46 \mathrm{GHz}$. In that case, $B=1.7 \mu \mathrm{G}$ and $\delta=$ 4.8. The magnetic field is an upper limit and the Doppler factor a lower limit, since the radio flux is just an upper limit. The lower limit to $\delta$ implies that the jet is within $12^{\circ}$ of our line of sight and therefore at least $670 \mathrm{kpc}$ in length. For comparison, if $f_{\nu}$ were $20 \mu \mathrm{Jy}$, we would have $B=0.65 \mu \mathrm{G}$ and $\delta=6.8$. Since we do not resolve the jet, it could be very much smaller. This would cause both $B$ and $\delta$ to have larger values than the numbers quoted. In any case the $(B, \delta)$ point must lie to the left and above the upward slanting solid line in Figure 4 and to the right and above a line joining the points where the two solid and two dashed lines intersect.

Electrons with $\gamma=1000 / \Gamma$ produce $\approx 1 \mathrm{keV}$ X-rays when Compton scattering off the microwave background. Such electrons will produce synchrotron radiation at too low a frequency to be observed if $B \lesssim 10 \Gamma^{2} \mu \mathrm{G}$. So an alternate explanation for the observed lack of a radio jet is that the electron spectrum breaks, e.g., because of aging. If the radio break is at $1 \mathrm{GHz}$ and $B=1.7 \mu \mathrm{G}$, the electron spectrum breaks at a Lorentz factor $\leq 10^{4}$. The lifetime of $\gamma=10^{4}$ electons against Compton scattering on the CMB at $z=1.866$ is about $3.6 \Gamma^{-2} \times 10^{6} \mathrm{yr}$.

\section{ALTERNATE INTERPRETATIONS}

Some faint galaxies, $r^{\prime}=23-24$, can be seen more or less overlapping the region of the western X-ray contours in Figure 1. They are much too faint to expect that normal galactic emission provides the X-rays, and the positions cannot be associated with the X-ray emission peaks, especially after adjusting the X-ray contours to coincide with the QSO. Both these objections could be overcome if these objects are a cluster of active galaxies.

Another possibility would be a foreground group of galaxies, at very much lower redshift. This requires only a single un- 
related source to be superposed near the QSO by chance. Bauer et al. (2002) reports a density of extended sources at this flux level to be $\approx 10 \mathrm{deg}^{-2}$, so there would be a $0.2 \%$ chance of such a source at this location. Since the ChaMP project expects to study several thousand sources, such a situation may occur. However, it would be strange that the X-rays do not center on the obvious $z=0.32$ galaxy 8 " to the north. The X-ray shape is quite distorted, so we would be viewing the cluster in an active and interesting dynamical state. The cluster might be involved in gravitational lensing of the QSO. We might have a failed cluster (Tucker et al. 1995) with only hot gas and no galaxy formation. In case of a foreground cluster, if hot gas overlaps the QSO position, future large throughput spectroscopy might use the Krolik \& Raymond (1988) test to measure angular diameter distance independently of redshift. Any of these possibilities would result in J0841 being a very exciting system.

\section{CONCLUSIONS}

Schwartz (2002b) has noted that X-ray emission by IC/CMB should result in X-ray jets being cosmic beacons-maintaining the same surface brightness at any larger redshift. This is because the $(1+z)^{-4}$ cosmic diminution of surface brightness is exactly compensated by the $(1+z)^{4}$ increase in the energy density of the $\mathrm{CMB}$ with redshift. Such an effect does not depend on equipartition or on relativistic beaming.
The low magnetic field, $\leq 2 \mu \mathrm{G}$, implied by the limits to radio emission is unusual. Fields in clusters of galaxies can approach $1 \mu \mathrm{G}$, while typical jet fields on kiloparsec scales are of the order of $10 \mu \mathrm{G}$. So the upper limits to magnetic field strengths derived here are somewhat weak for a jet. However, there seems to be no fundamental physics prohibiting massive black holes to produce jets of such low internal energy density. Selection bias against finding radio-quiet X-ray jets could explain why such low magnetic field jets have not previously been noted. Alternately, this object may have a magnetic field much weaker than the equipartition value.

This work was supported in part by NASA contract NAS839073 to the Chandra X-ray Center (CXC) and CXC grants AR2-3009X and GO2-3151C to SAO. We thank D. Harris for discussions and for comments on the manuscript and D. Jerius for assistance with telescope coordinate systems and the raytrace results. This research used the NASA Astrophysics Data System Bibliographic Services and the NASA/IPAC Extragalactic Database, which is operated by the Jet Propulsion Laboratory, California Institute of Technology, under contract with NASA. We thank the VLA for the allocation of $1 \mathrm{hr}$ of discretionary time. The National Radio Astronomy Observatory is a facility of the National Science Foundation operated under cooperative agreement by Associated Universities, Inc.

\section{REFERENCES}

Bauer, F. E., et al. 2002, AJ, 123, 1163

Brunetti, G., Bondi, M., Comastri, A., \& Setti, G. 2002, A\&A, 381, 795

Celotti, A., Ghisellini, G., \& Chiaberge, M. 2001, MNRAS, 321, L1

Dermer, C. D., \& Schlickeiser, R. 1994, ApJS, 90, 945

Giacconi, R., et al. 2001, ApJ, 551, 624

Green, P. J., et al. 2004, ApJS, 150, 43

Harris, D. E., \& Krawczynski, H. 2002, ApJ, 565, 244

Kim, D.-W., et al. 2004, ApJS, 150, 19

Krolik, J. H., \& Raymond, J. C. 1988, ApJ, 335, L39

Maccacaro, T., della Ceca, R., Gioia, I. M., Morris, S. L., Stocke, J. T., \& Wolter, A. 1991, ApJ, 374, 117

Marshall, H. L., et al. 2001, ApJ, 549, L167

Sambruna, R. M., Urry, C. M., Tavecchio, F., Maraschi, L., Scarpa, R., Chartas, G., \& Muxlow, T. 2001, ApJ, 549, L161
Schwartz, D. A. 2002a, in Lighthouses of the Universe: The Most Celestial Objects and Their Use for Cosmology, ed. M. Gilfanov, R. A. Siuniaev, \& E. Churazov (Berlin: Springer), 538 . 2002b, ApJ, 569, L23

Schwartz, D. A., et al. 2000, ApJ, 540, L69

Siemiginowska, A, Bechtold, J., Aldcroft, T. L., Elvis, M., Harris, D. E., \& Dobrzycki, A. 2002, ApJ, 570, 543

Stark, A. A., Gammie, C. F., Wilson, R. W., Bally, J., Linke, R. A., Heiles, C., \& Hurwitz, M. 1992, ApJS, 79, 77

Tavecchio, F., et al. 2000, ApJ, 544, L23

Tucker, W. H., Tananbaum, H., \& Remillard, R. A. 1995, ApJ, 444, 532

Vanden Berk, D. E. 2001, AJ, 122, 549 